\title{
A Korean-American Comparative Study of 3D Scanned Female Anthropometric Data
}

\author{
Yi Kyonghwa* ${ }^{*}$ Cynthia L. Istook \\ Professor, Dept. of Clothing \& Textiles, The Catholic University of Korea* \\ Professor, Dept. of Textile Apparel Technology \& Management, \\ North Carolina State University
}

\begin{abstract}
The purpose of this research is to provide useable data for application in American and Korean apparel company. This data was developed by analyzing information of Korean and American body sizes obtained from "Size USA Project" and "Size Korea Project".

The Subjects were 6,306 American females and 1,988 Korean females over 18 years old. 30 measurements and 14 computed values were chosen that were considered critical in making garments. And descriptive analysis, percentile analysis and t-test were used as statistical methods for analyzing measurements and computed value between the two countries.

The results were as follows. It was determined that American women were larger and bigger than Korean women in all measurements and computed values, except for Shoulder Slope. Based on BMl values, we determined that American women had a distinct tendency towards being overweight.

Through the comparison of drop values (i.e. the difference between Hip and Bust Girths or Hip and Waist Girths), ratio values (i.e. waist height divided by height) and Body Mass Index (BMI) between the two countries, we determined that American women's figures were shapelier than Korean women's. American women had higher hip heights and longer leg lengths for their height compared to Korean woman. Furthermore, the back shapes of Korean women were flatter than American women and BMl values indicated American women were relatively more overweight than Korean women.
\end{abstract}

Key Words : 3D body scanning, anthropometric, body measurements, laser scanners, CAD

\footnotetext{
+ This work was supported by the Catholic University of Korea, Research Fund, 2013

Size USA measurement data were provided by $[\mathrm{TC}]^{2}$. And Size Korea measurement data were provided by Korean Agency for Technology \& Standards.
}

Corresponding author: Yi Kyonghwa, Tel. +82-2-2164-4329, Fax. +82-2-2164-4320

E-mail:ykh@catholic.ac.kr 


\section{Introduction}

Traditionally, body size and shape has been viewed using ratios such as 36-26-36, which correspond to actual body measurements at the locations of bust-waist-hip. However, university researchers, apparel producers, and consumers are beginning to understand that the numbers themselves are not as important as the ratio between these numbers, and the body proportions they represent. Anthropometric research using body scan data provides information on consumer body sizes and shapes to designers and manufacturers to improve the fit of garments. ${ }^{1}$

Size USA Project (7/2002-9/2003), collected body measurement and demographic data of over 10,000 subjects were collected in 13 cities. Historically, almost all U.S. sizing surveys conducted were for the U.S. military for the purpose of sizing equipment and military apparel. ${ }^{2)}$ Except for Size USA survey, a comprehensive study of the size and shape distributions of the United States civilian population has never been done. $^{3)}$

A few precedent researches about body sizes and shape analysis of American women were done by using this Size USA 3D scanned data since 2004. But until now, there were no researches about international comparative study between the U.S. and other country.

Korea also completed an international comparative study of body sizes and established several apparel sizing standards ${ }^{45)}$ in 2005 as a "Post Size Korea Study".6) But the information was limited due to the lack of recent and up to date research data on body sizes.

In this paper, we analyzed 3D scanned anthropometric measurement data of 6306 American female adults 18 years old and more from Size USA and 1988 Korean female adults 18 years old and more from Size Korea. We used various statistical methods such as, descriptive analysis, percentile analysis and t-test between the two countries' measurements. And we also analyzed drop values, ratios and for understanding of physical characteristics.

\section{Methodology}

The purpose of this study was to compare the body sizes and shapes of Korean females and American females.

The brief comparison of characteristics in Size USA and Size Korea was shown in 〈Table 1> and demographic distribution of the subjects in two countries was shown as $\langle$ Table 2>.

30 measurements and 14 computed values were chosen that were considered critical in making garments. These measurements used in this paper showed in <Table 3>. These measurements were all standardized international measurements methods came from $\mid S \mathrm{SO}^{7 / 8)(9) 10 \text {, }}$ ASTM $^{(1)(12)(3) 14115)}$ and KS. ${ }^{16)}$

The $\mathrm{t}$-test between American \& Korean females' main measurements by bust groups according to ASTM D 5585 were fulfilled because this research data, which is one of the initial steps to make a sizing conversion table between the two countries will contribute to the continuing effort to standardize import apparel sizing for the Korean market. 
$<$ Table $1>$ Comparison of Size USA \& Size Korea

\begin{tabular}{|c|c|c|}
\hline & Size USA & Size Korea \\
\hline Period & 2002 2003 & 2003 2004 \\
\hline Scanner & {$[\mathrm{TC}]^{2}$} & Cyberware \\
\hline Software & $\begin{array}{l}\text { Body Measurement System } \\
\text { (Visual basic/C } \mathrm{C}^{++} / \text {open GL Graphics) }\end{array}$ & $\begin{array}{l}\text { Editing S/W: RapidForm } 2004 \\
\text { Measurement S/W: 3DM }\end{array}$ \\
\hline Subjects & $\begin{array}{l}\text { 1) } 6 \text { age groups (18-25, 26-35, 36-45, } \\
46-55,56-65,66+) \\
\text { 2) Female: } 6,310 \\
\text { 3) } 4 \text { ethnic groups (White, Black, Hispanic } \\
\text { and Other) } \\
\text { * Note: Asian subjects are included in } \\
\text { Other. }\end{array}$ & $\begin{array}{l}\text { 1) 18 75 years old } \\
\text { 2) Female: } 1,988 \\
\text { * Note: Analyzed fixed ages only and } \\
\text { adjusted age groups same to USA }\end{array}$ \\
\hline $\begin{array}{l}\text { Measurement } \\
\text { variables }\end{array}$ & Over 140 automatic body measurements & $\begin{array}{l}205 \text { total measured, includes } 126 \text { body } \\
\text { measurements, } 79 \text { Foot/head } \\
\text { measurements }\end{array}$ \\
\hline
\end{tabular}

$<$ Table 2> Demographic distribution of the subjects

\begin{tabular}{|c|c|c|c|c|c|c|c|c|c|c|c|c|}
\hline \multirow{3}{*}{$\begin{array}{l}\text { Age } \\
\text { group }\end{array}$} & \multirow{2}{*}{\multicolumn{2}{|c|}{ Korean }} & \multicolumn{10}{|c|}{ American } \\
\hline & & & \multicolumn{2}{|c|}{ White } & \multicolumn{2}{|c|}{ Black } & \multicolumn{2}{|c|}{ Hispanic } & \multicolumn{2}{|c|}{ Others } & \multicolumn{2}{|c|}{ Group Total } \\
\hline & Count & $\%$ & Count & $\%$ & Count & $\%$ & Count & $\%$ & Count & $\%$ & Count & $\%$ \\
\hline $18 \sim 25$ & 463 & 23.3 & 707 & 1.2 & 293 & 4.6 & 271 & 4.3 & 266 & 4.2 & 1537 & 24.4 \\
\hline $26 \sim 35$ & 518 & 26.1 & 653 & 10.4 & 290 & 4.6 & 233 & 3.7 & 271 & 4.3 & 1447 & 22.9 \\
\hline $36 \sim 45$ & 382 & 19.2 & 686 & 10.9 & 268 & 4.2 & 176 & 2.8 & 210 & 3.3 & 1340 & 21.2 \\
\hline $46 \sim 55$ & 272 & 13.7 & 682 & 10.8 & 194 & 3.1 & 106 & 1.7 & 159 & 2.5 & 1141 & 18.1 \\
\hline $56 \sim 65$ & 194 & 9.8 & 413 & 6.5 & 54 & .9 & 44 & .7 & 94 & 1.5 & 605 & 9.6 \\
\hline $66 \sim$ & 159 & 8.0 & 187 & 3.0 & 6 & .1 & 8 & .1 & 35 & .6 & 236 & 3.7 \\
\hline $\begin{array}{c}\text { Group } \\
\text { Total } \\
\end{array}$ & 1988 & 100.0 & 3328 & 52.8 & 1105 & 17.5 & 838 & 13.3 & 1035 & 16.4 & 6306 & 100.0 \\
\hline
\end{tabular}

\section{Results}

\section{American/Korean analysis of the body sizes}

The percentile values of America and Korean females were as $\langle$ Table 4$\rangle$. In most of the measurements, the means and each percentile of American females were bigger than Korean females. And the measurement ranges of American females were wider than Korean females except for "Shoulder Slope".

$<$ Table 5> was the results showing differences of body sizes between the two countries. All measurements except "Crotch Length Total" were different significantly between Americans and Koreans. All Heights measurement variables of 
Yi Kyonghwa - Cynthia L. Istook / A Korean-American Comparative Study of 3D Scanned Female Anthropometric Data

<Table 3> Measurements and Computed value were used

\begin{tabular}{|c|c|c|c|}
\hline & Measurements & & Measurements \\
\hline \multirow{13}{*}{ Girths } & 1. Bust Girth & \multirow{5}{*}{ Heights } & 16. Waist Height \\
\hline & 2. Waist Girth & & 17. Hip Height \\
\hline & 3. Hip Girth & & 18. Crotch Height \\
\hline & 4. Mid-Neck Girth & & 19. Knee Height \\
\hline & 5. Neck Base Girth & & 20. Ankle Height \\
\hline & 6. Armscye Girth & \multirow{9}{*}{ Lengths } & 21. Waist Length (Front) \\
\hline & 7. Elbow Girth & & 22. Waist Length (Back) \\
\hline & 8. Thigh, Max Girth & & 23. Cross-Back Width(Interscye Fold, Back) \\
\hline & 9. Thigh, Mid Girth & & 24. Cross-Chest Width(Interscye Fold, Front) \\
\hline & 10. Knee Girth & & 25. Arm Length (Shoulder to Wrist) \\
\hline & 11. Calf Girth & & 26. Arm Length (Center Back Neck to Wrist) \\
\hline & 12. Ankle Girth & & 27. Bust Point to Bust Point \\
\hline & 13. Total Crotch Length & & 28. Side Neck to Bust Point \\
\hline \multirow{3}{*}{ Heights } & 14. Height & & 29. Shoulder Slope(degree) \\
\hline & 15. Back Neck Point Height & Others & 30. Weight(Kg) \\
\hline & Computed values & & Computed values \\
\hline \multirow{3}{*}{$\begin{array}{c}\text { Drop } \\
\text { Values }\end{array}$} & C1. Hip girth-Bust girth & \multirow{6}{*}{ Ratios } & C8. Knee Height/Height \\
\hline & C2. Hip girth -Waist girth & & C9. Ankle Height/Height \\
\hline & C3. Bust girth -Waist girth & & C10. Waist Length Front/Height \\
\hline \multirow{4}{*}{ Ratios } & C4. Back Neck Point/Height & & C11. Waist Length Back/Height \\
\hline & C5. Waist Height/Height & & C12. Waist/height \\
\hline & C6. Hip Height/Height & & C13. Hip /Height \\
\hline & C7. Crotch Height/Height & Index & C14. BMI \\
\hline
\end{tabular}

$\mathrm{BMl}=$ weight $(\mathrm{Kg}) /$ height $\left(\mathrm{m}^{2}\right)$

American females were higher than those of Korean females. All Girth measurements of American females were bigger than those of Korean females. In case of Lengths measurements, American females' measurements were longer than Korean females' measurements. "Shoulder Slopes (degrees)" of American females is smaller than Korean females. We concluded American women had "risen shoulder" when compared with Korean women. In terms of weight, American females were also heavier than Korean females.
$<$ Table 6> showed Korean Standard \% values when American measurements were considered as $100 \%$.

In case Korean Standard \% values were higher than those of American, each value was highlighted. Korean women's value was higher than American in shoulder slope measurement. But most values of American females were higher than Korean females. According to analysis of standard values of American vs. Korean females, Korean women had smaller 
Journal of Fashion Business Vol.17, No.3

$<$ Table 4> American/Korean Percentile analysis of Measurements

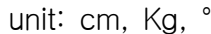

\begin{tabular}{|c|c|c|c|c|c|c|c|c|}
\hline Measurements & Country & Mean & $\begin{array}{c}\% \text { tile } \\
05\end{array}$ & $\begin{array}{c}\% \text { tile } \\
25\end{array}$ & $\begin{array}{c}\% \text { tile } \\
50\end{array}$ & $\begin{array}{c}\% \text { tile } \\
75\end{array}$ & $\begin{array}{c}\% \text { tile } \\
95\end{array}$ & Range* \\
\hline \multirow{2}{*}{ Bust Girth } & U.S. & 103.67 & 85.84 & 94.28 & 101.82 & 111.27 & 127.83 & 41.99 \\
\hline & Korea & 90.20 & 78.80 & 84.40 & 89.30 & 95.18 & 104.52 & 25.72 \\
\hline \multirow{2}{*}{ Waist Girth } & U.S. & 87.40 & 69.61 & 77.20 & 84.85 & 95.04 & 114.11 & 44.50 \\
\hline & Korea & 76.87 & 63.19 & 69.20 & 75.40 & 83.30 & 95.40 & 32.22 \\
\hline \multirow{2}{*}{ Hip Girth } & U.S. & 109.61 & 93.18 & 100.62 & 107.30 & 116.00 & 134.58 & 41.40 \\
\hline & Korea & 93.58 & 85.70 & 89.90 & 93.40 & 96.70 & 102.90 & 17.20 \\
\hline \multirow{2}{*}{ Mid Neck Girth } & U.S. & 35.77 & 31.22 & 33.21 & 35.14 & 37.70 & 42.46 & 11.24 \\
\hline & Korea & 32.74 & 28.60 & 30.80 & 32.40 & 34.50 & 37.60 & 9.00 \\
\hline \multirow{2}{*}{ Neck Base Girth } & U.S. & 38.32 & 33.95 & 35.97 & 37.86 & 40.14 & 44.33 & 10.38 \\
\hline & Korea & 37.69 & 33.39 & 35.60 & 37.60 & 39.60 & 42.50 & 9.12 \\
\hline \multirow{2}{*}{ Armscye Girth } & U.S. & 42.27 & 35.31 & 38.77 & 41.70 & 45.19 & 51.16 & 15.85 \\
\hline & Korea & 35.27 & 30.70 & 32.90 & 35.00 & 37.30 & 40.70 & 10.00 \\
\hline \multirow{2}{*}{ Elbow Girth } & U.S. & 26.39 & 22.40 & 24.22 & 25.86 & 27.96 & 32.12 & 9.72 \\
\hline & Korea & 23.16 & 19.60 & 21.70 & 23.20 & 24.60 & 26.82 & 7.22 \\
\hline \multirow{2}{*}{ Thigh Max Girth } & U.S. & 62.52 & 52.60 & 57.25 & 61.31 & 66.48 & 76.81 & 24.21 \\
\hline & Korea & 55.44 & 48.40 & 52.30 & 55.20 & 58.20 & 63.40 & 15.00 \\
\hline \multirow{2}{*}{ Thigh Mid Girth } & U.S. & 51.26 & 43.01 & 46.87 & 50.14 & 54.51 & 63.52 & 20.51 \\
\hline & Korea & 49.44 & 43.20 & 46.70 & 49.20 & 51.90 & 56.20 & 13.00 \\
\hline \multirow{2}{*}{ Knee Girth } & U.S. & 39.06 & 34.06 & 36.48 & 38.47 & 41.04 & 46.11 & 12.05 \\
\hline & Korea & 35.16 & 30.89 & 33.40 & 35.10 & 36.90 & 39.40 & 8.52 \\
\hline \multirow{2}{*}{ Calf Girth } & U.S. & 38.14 & 32.82 & 35.36 & 37.62 & 40.21 & 45.54 & 12.73 \\
\hline & Korea & 34.09 & 29.60 & 32.20 & 34.00 & 35.90 & 38.72 & 9.12 \\
\hline \multirow{2}{*}{ Ankle Girth } & U.S. & 25.58 & 22.60 & 24.03 & 25.18 & 26.65 & 30.11 & 7.51 \\
\hline & Korea & 23.57 & 20.20 & 22.20 & 23.50 & 25.00 & 27.10 & 6.90 \\
\hline \multirow{2}{*}{ Crotch Length Total } & U.S. & 72.40 & 60.09 & 66.55 & 71.23 & 76.73 & 89.82 & 29.73 \\
\hline & Korea & 72.17 & 65.20 & 69.20 & 72.20 & 75.00 & 79.30 & 14.10 \\
\hline \multirow{2}{*}{ Height } & U.S. & 162.74 & 151.55 & 157.91 & 163.01 & 166.83 & 174.47 & 22.92 \\
\hline & Korea & 157.37 & 147.66 & 153.40 & 157.30 & 161.30 & 167.40 & 19.74 \\
\hline \multirow{2}{*}{$\begin{array}{l}\text { Back Neck Point } \\
\text { Height }\end{array}$} & U.S. & 139.85 & 129.52 & 135.43 & 139.79 & 144.05 & 150.28 & 20.76 \\
\hline & Korea & 132.71 & 124.00 & 129.10 & 132.60 & 136.20 & 141.70 & 17.70 \\
\hline \multirow{2}{*}{ Waist Height } & U.S. & 100.29 & 90.67 & 96.13 & 100.23 & 104.36 & 110.44 & 19.76 \\
\hline & Korea & 96.94 & 89.00 & 93.50 & 97.00 & 100.20 & 105.00 & 16.00 \\
\hline \multirow{2}{*}{ Hip Height } & U.S. & 81.37 & 71.13 & 76.47 & 80.77 & 85.87 & 93.61 & 22.48 \\
\hline & Korea & 75.61 & 69.10 & 72.90 & 75.50 & 78.30 & 82.40 & 13.30 \\
\hline \multirow{2}{*}{ Crotch Height } & U.S. & 73.42 & 65.74 & 70.20 & 73.34 & 76.58 & 81.36 & 15.62 \\
\hline & Korea & 70.02 & 63.90 & 67.40 & 69.90 & 72.50 & 76.40 & 12.50 \\
\hline \multirow{2}{*}{ Knee Height } & U.S. & 44.31 & 39.67 & 42.41 & 44.34 & 46.20 & 49.15 & 9.48 \\
\hline & Korea & 40.22 & 36.90 & 38.80 & 40.20 & 41.60 & 43.70 & 6.80 \\
\hline \multirow{2}{*}{ Ankle Height } & U.S. & 6.98 & 5.26 & 6.27 & 7.02 & 7.52 & 8.52 & 3.26 \\
\hline & Korea & 5.31 & 4.50 & 4.90 & 5.30 & 5.70 & 6.20 & 1.70 \\
\hline
\end{tabular}

* Note: Range=Percentile 95-Percentile 05 
Yi Kyonghwa - Cynthia L. Istook / A Korean-American Comparative Study of 3D Scanned

Female Anthropometric Data

\section{$<$ Table 4> Continued}

unit: $\mathrm{cm}, \mathrm{Kg}$,

\begin{tabular}{|c|c|c|c|c|c|c|c|c|}
\hline Measurements & Country & Mean & $\begin{array}{l}\text { \%tile } \\
05\end{array}$ & $\begin{array}{c}\text { \%tile } \\
25\end{array}$ & $\begin{array}{c}\text { \%tile } \\
50\end{array}$ & $\begin{array}{l}\text { \%tile } \\
75\end{array}$ & $\begin{array}{l}\text { \%tile } \\
95\end{array}$ & Range* \\
\hline \multirow{2}{*}{ Waist Length_F } & U.S. & 37.57 & 31.28 & 34.68 & 37.13 & 40.11 & 45.24 & 13.96 \\
\hline & Korea & 32.38 & 29.00 & 30.80 & 32.30 & 33.90 & 36.20 & 7.20 \\
\hline \multirow{2}{*}{ Waist Length-B } & U.S. & 44.01 & 39.22 & 42.02 & 43.93 & 45.91 & 49.04 & 9.82 \\
\hline & Korea & 38.41 & 35.10 & 36.93 & 38.30 & 39.90 & 41.92 & 6.82 \\
\hline \multirow{2}{*}{ Cross Back Width } & U.S. & 36.75 & 31.25 & 34.22 & 36.39 & 38.87 & 43.40 & 12.15 \\
\hline & Korea & 35.34 & 31.60 & 33.70 & 35.20 & 36.80 & 39.52 & 7.92 \\
\hline \multirow{2}{*}{ Cross Chest Width } & U.S. & 37.75 & 29.73 & 34.73 & 37.64 & 40.74 & 45.90 & 16.18 \\
\hline & Korea & 33.79 & 30.40 & 32.30 & 33.70 & 35.10 & 37.60 & 7.20 \\
\hline \multirow{2}{*}{$\begin{array}{l}\text { Arm Length } \\
\text { (Shoulder to Wrist) }\end{array}$} & U.S. & 53.54 & 47.31 & 51.07 & 53.47 & 55.98 & 59.83 & 12.53 \\
\hline & Korea & 50.77 & 47.00 & 49.10 & 50.70 & 52.40 & 54.60 & 7.60 \\
\hline \multirow{2}{*}{$\begin{array}{l}\text { Arm Length } \\
\text { (CB Neck to Wrist) }\end{array}$} & U.S. & 72.64 & 65.39 & 69.70 & 72.55 & 75.48 & 80.05 & 14.65 \\
\hline & Korea & 71.38 & 66.60 & 69.40 & 71.30 & 73.30 & 76.30 & 9.70 \\
\hline \multirow{2}{*}{ Bust Pt to Bust Pt } & U.S. & 21.04 & 17.37 & 19.47 & 20.85 & 22.36 & 25.51 & 8.14 \\
\hline & Korea & 15.62 & 13.40 & 14.60 & 15.60 & 16.50 & 18.10 & 4.70 \\
\hline \multirow{2}{*}{ Side Neck to Bust Pt } & U.S. & 27.97 & 23.53 & 25.89 & 27.69 & 29.81 & 33.47 & 9.94 \\
\hline & Korea & 26.24 & 22.70 & 24.50 & 26.10 & 27.70 & 30.50 & 7.80 \\
\hline \multirow{2}{*}{$\begin{array}{l}\text { Shoulder Slope } \\
\text { (degrees) }\end{array}$} & U.S. & 21.22 & 14.97 & 18.64 & 21.26 & 23.83 & 27.27 & 12.31 \\
\hline & Korea & 24.68 & 18.00 & 22.00 & 25.00 & 27.00 & 31.00 & 13.00 \\
\hline \multirow{2}{*}{$\begin{array}{l}\text { Weight } \\
(\mathrm{Kg})\end{array}$} & U.S. & 70.57 & 48.98 & 58.50 & 66.66 & 78.91 & 104.76 & 55.78 \\
\hline & Korea & 56.32 & 44.50 & 51.00 & 55.50 & 61.00 & 71.00 & 26.50 \\
\hline
\end{tabular}

* Note: Range=Percentile 95-Percentile 05

$\left\langle\right.$ Table 5> American/Korean differences of the body sizes ${ }^{17)}$

unit: $\mathrm{cm}, \mathrm{Kg}$,

\begin{tabular}{|c|c|c|c|c|c|c|}
\hline \multirow{2}{*}{ Measurements } & \multicolumn{2}{|c|}{ US } & \multicolumn{2}{|c|}{ Korean } & \multirow{2}{*}{$t$} & \multirow{2}{*}{ Sig. } \\
\hline & Mean & S.D. & Mean & S.D. & & \\
\hline Bust Girth & 103.67 & 12.85 & 89.88 & 9.45 & 44.12 & 0.000 \\
\hline Waist Girth & 87.40 & 13.83 & 76.60 & 10.87 & 31.40 & 0.000 \\
\hline Hip Girth & 109.61 & 12.75 & 93.01 & 9.02 & 54.33 & 0.000 \\
\hline Mid-Neck Girth & 35.77 & 3.51 & 32.62 & 3.35 & 35.15 & 0.000 \\
\hline Neck Base Girth & 38.32 & 3.21 & 37.55 & 3.62 & 7.86 & 0.000 \\
\hline Armscye Girth & 42.27 & 5.11 & 35.14 & 3.73 & 57.64 & 0.000 \\
\hline Elbow Girth & 26.39 & 3.20 & 23.16 & 2.19 & 41.88 & 0.000 \\
\hline Thigh Max Girth & 62.52 & 7.49 & 55.24 & 5.65 & 39.73 & 0.000 \\
\hline Thigh Mid Girth & 51.26 & 6.35 & 49.27 & 5.04 & 11.95 & 0.000 \\
\hline Knee Girth & 39.06 & 3.80 & 35.04 & 3.40 & 42.37 & 0.000 \\
\hline Calf Girth & 38.14 & 4.00 & 33.97 & 3.45 & 41.95 & 0.000 \\
\hline Ankle Girth & 25.58 & 2.31 & 23.49 & 2.49 & 34.39 & 0.000 \\
\hline Crotch Length Total & 72.40 & 9.02 & 71.92 & 6.05 & 1.09 & 0.277 \\
\hline Height & 162.74 & 6.98 & 156.42 & 13.57 & 30.81 & 0.000 \\
\hline
\end{tabular}


$<$ Table 5> Continued

unit: $\mathrm{cm}, \mathrm{Kg},{ }^{\circ}$

\begin{tabular}{l|r|r|r|r|r|r}
\hline \multirow{2}{*}{ Measurements } & \multicolumn{2}{c|}{ US } & \multicolumn{2}{c|}{ Korean } & \multirow{2}{*}{ t } & \multirow{2}{*}{ Sig. } \\
\cline { 2 - 6 } & \multicolumn{1}{c|}{ Mean } & \multicolumn{1}{c|}{ S.D. } & \multicolumn{1}{c|}{ Mean } & \multicolumn{1}{c}{ S.D. } & & \multicolumn{1}{c}{. } \\
\hline Back Neck Point Height & 139.85 & 6.37 & 131.91 & 11.58 & 45.02 & 0.000 \\
\hline Waist Height & 100.29 & 6.00 & 96.36 & 8.94 & 22.58 & 0.000 \\
\hline Hip Height & 81.37 & 6.81 & 75.15 & 7.10 & 35.68 & 0.000 \\
\hline Crotch Height & 73.42 & 4.77 & 69.59 & 6.66 & 28.85 & 0.000 \\
\hline Knee Height & 44.31 & 3.04 & 39.97 & 3.75 & 55.78 & 0.000 \\
\hline Ankle Height & 6.98 & 1.00 & 5.28 & 0.68 & 70.73 & 0.000 \\
\hline Waist Length Front & 37.57 & 4.23 & 32.27 & 2.91 & 52.33 & 0.000 \\
\hline Waist Length Back & 44.01 & 2.99 & 38.28 & 3.14 & 77.11 & 0.000 \\
\hline Cross Back Width & 36.75 & 3.72 & 35.22 & 3.18 & 15.86 & 0.000 \\
\hline Cross Chest Width & 37.75 & 4.94 & 33.66 & 3.08 & 34.49 & 0.000 \\
\hline Arm Length(Shoulder to Wrist) & 53.54 & 3.86 & 50.59 & 3.82 & 30.11 & 0.000 \\
\hline Arm Length CB Neck to Wrist & 72.64 & 4.47 & 71.13 & 5.13 & 11.79 & 0.000 \\
\hline Bust Pt to Bust Pt & 21.04 & 2.41 & 15.56 & 1.69 & 95.14 & 0.000 \\
\hline Side Neck to Bust Point & 27.97 & 3.03 & 26.15 & 2.83 & 23.29 & 0.000 \\
\hline Shoulder Slope(degrees) & 21.22 & 3.75 & 24.68 & 4.12 & -34.95 & 0.000 \\
\hline Weight(Kg) & 70.57 & 17.43 & 56.32 & 7.88 & 35.30 & 0.000 \\
\hline \hline
\end{tabular}

* The part of this table was cited from the original paper of Yi, Kyong-Hwa, et. al. in 2007 ${ }^{17)}$

$<$ Table 6> Standard values of American females vs. Korean females

Unit: \%

\begin{tabular}{l|r|r|l|r|r}
\hline \hline \multicolumn{1}{c|}{ Measurements } & \multicolumn{1}{c|}{ US } & \multicolumn{1}{c|}{ Korean } & \multicolumn{1}{c|}{ Measurements } & US & Korean \\
\hline Bust Girth & 100.0 & 86.7 & Waist Height & 100.0 & 96.1 \\
\hline Waist Girth & 100.0 & 87.6 & Hip Height & 100.0 & 92.4 \\
\hline Hip Girth & 100.0 & 84.9 & Crotch Height & 100.0 & 94.8 \\
\hline Mid-Neck Girth & 100.0 & 91.2 & Knee Height & 100.0 & 90.2 \\
\hline Neck Base Girth & 100.0 & 98.0 & Ankle Height & 100.0 & 75.6 \\
\hline Armscye Girth & 100.0 & 83.1 & Waist Length Front & 100.0 & 85.9 \\
\hline Elbow Girth & 100.0 & 87.8 & Waist Length Back & 100.0 & 87.0 \\
\hline Thigh Max Girth & 100.0 & 88.4 & Cross Back Width & 100.0 & 95.8 \\
\hline Thigh Mid Girth & 100.0 & 96.1 & Cross Chest Width & 100.0 & 89.2 \\
\hline Knee Girth & 100.0 & 89.7 & Shoulder Slope(degrees) & 100.0 & 116.3 \\
\hline Calf Girth & 100.0 & 89.1 & Weight & 100.0 & 79.8 \\
\hline Ankle Girth & 100.0 & 91.8 & Arm Length(Shoulder to Wrist) & 100.0 & 94.5 \\
\hline Crotch Length Total & 100.0 & 99.3 & Arm Length(CB Neck to Wrist) & 100.0 & 97.9 \\
\hline Height & 100.0 & 96.1 & Bust Pt to Bust Pt & 100.0 & 74.0 \\
\hline Back Neck Point Height & 100.0 & 94.3 & Side Neck to Bust Point & 100.0 & 93.5 \\
\hline \hline
\end{tabular}


girths, shorter heights and lengths, "sagged shoulders" compared with American women. Korean women's hip sizes were respectively smaller than American because hip values were the most low among the girth measurements. And Korean women's "Hip Height" was lower than American according to standard values. It has also been determined from <Table 6>.

1) Korean women's weight was $79.8 \%$ of American women's value, we can verify American women's obesity, 2) Korean women were lower than American women in the distance of "Bust Point to Bust Point". Namely, we can presume the possibility that American women had "wider and larger breast" compared with Korean women.

\section{American/Korean differences Analysis of drop values, ratios and $\mathrm{BMI}$}

When we compared the measurements between two groups and more, drop values, ratios and indices are very useful to understand the characteristics of body shapes. In this paper, Drop values such as "Hip Girth-Bust Girth", "Hip Girth-Waist Girth", "Bust Girth-waist Girth" were computed and analyzed. In case of ratios, main Height measurements for "Height" were computed and analyzed for understanding the proportion of body heights. BMI was used to verify degree of obesity. Body mass index (BMI) is a measurement of bodyfat based on height and weight that applies to both adult men and women

The computed measurements' percentile values of America and Korean females were as $\langle$ Table 7$\rangle$. All computed measurements except for "Waist Height/Height", the mean and each percentile value of American women were bigger or higher than Korean women. In case of range value which means range from percentile 05 to percentile 95.

Table 8 was the results showing differences of "Drop Values", Body ratios and BMl between the two countries. All Drop Values of American women were bigger than those of Korean women. These results intimated American women's "Hip Girths" and "Bust Girths" were large compared "Waist Girths". These also showed American women's body figures were more curved than Korean women. When "Hip Girth-Bust Girth" of American women were compared to "ISO/TR 10652 : 1991』, the mean drop value of American women's was just same as the mean drop value of "body type M" by ISO. But the mean drop value of Korean women's was close to the drop value of "body type $\mathrm{H}^{\prime \prime}$ by ISO.

According to other Height ratios by Height, "Back Neck Point Height", "Hip Height", "Crotch Height", "Knee Height", "Ankle Height" of Americans were statistically different from those of Koreans. But in case of "Waist Height/ Height", there was no significant difference between the two countries. In summary, we concluded American women had slightly short head length, risen hips and long leg length for their height compared Korean woman.

From BMI, we also concluded American women reached overweight category. The mean value of Americans' BMl showed 0.27 which indicates "Overweight category" while the mean value of Koreans' BMl showed below 0.25 which means "Normal weight".

In addition "Waist Girth/Height" and "Hip Girth/Height" showed the relative obesity of American women for Korean women. We also verified that American women's obesity tendency in these two values. 
$<$ Table $7>$ American/Korean Percentile analysis of Measurements

\begin{tabular}{|c|c|c|c|c|c|c|c|c|}
\hline Computed values & Country & Mean & $\begin{array}{c}\text { \%tile } \\
05\end{array}$ & $\begin{array}{c}\% \text { \%tile } \\
25\end{array}$ & $\begin{array}{c}\text { \%tile } \\
50\end{array}$ & $\begin{array}{c}\text { \%tile } \\
75\end{array}$ & $\begin{array}{c}\text { \%tile } \\
95\end{array}$ & Range* \\
\hline \multirow{2}{*}{ Hip Girth - Bust Girth } & U.S. & 5.94 & -4.88 & 1.56 & 5.83 & 10.14 & 16.98 & 21.86 \\
\hline & Korea & 3.14 & -7.60 & -0.50 & 4.20 & 7.70 & 12.40 & 20.00 \\
\hline \multirow{2}{*}{ Hip Girth - Waist Girth } & U.S. & 22.21 & 12.14 & 18.13 & 22.34 & 26.25 & 32.17 & 20.03 \\
\hline & Korea & 16.41 & 0.90 & 11.80 & 18.40 & 22.90 & 27.30 & 26.40 \\
\hline \multirow{2}{*}{ Bust Girth - Waist Girth } & U.S. & 16.27 & 8.34 & 13.13 & 16.35 & 19.45 & 24.09 & 15.76 \\
\hline & Korea & 13.33 & 5.98 & 10.80 & 13.80 & 16.00 & 19.30 & 13.32 \\
\hline \multirow{2}{*}{$\begin{array}{l}\text { Back Neck Point } \\
\text { Height/Height }\end{array}$} & U.S. & 0.86 & 0.84 & 0.85 & 0.86 & 0.87 & 0.87 & 0.03 \\
\hline & Korea & 0.84 & 0.83 & 0.84 & 0.84 & 0.85 & 0.85 & 0.02 \\
\hline \multirow{2}{*}{ Waist Height/Height } & U.S. & 0.62 & 0.58 & 0.60 & 0.62 & 0.63 & 0.65 & 0.07 \\
\hline & Korea & 0.62 & 0.59 & 0.61 & 0.62 & 0.62 & 0.64 & 0.04 \\
\hline \multirow{2}{*}{ Hip Height/Height } & U.S. & 0.50 & 0.45 & 0.47 & 0.49 & 0.53 & 0.56 & 0.11 \\
\hline & Korea & 0.48 & 0.46 & 0.47 & 0.48 & 0.49 & 0.50 & 0.04 \\
\hline \multirow{2}{*}{ Crotch Height/Heigh } & U.S. & 0.45 & 0.42 & 0.44 & 0.45 & 0.46 & 0.48 & 0.05 \\
\hline & Korea & 0.44 & 0.42 & 0.44 & 0.44 & 0.45 & 0.47 & 0.04 \\
\hline \multirow{2}{*}{ Knee Height/Height } & U.S. & 0.27 & 0.26 & 0.27 & 0.27 & 0.28 & 0.29 & 0.03 \\
\hline & Korea & 0.26 & 0.24 & 0.25 & 0.26 & 0.26 & 0.27 & 0.02 \\
\hline \multirow{2}{*}{ Ankle Height/Height } & U.S. & 0.04 & 0.03 & 0.04 & 0.04 & 0.05 & 0.05 & 0.02 \\
\hline & Korea & 0.03 & 0.03 & 0.03 & 0.03 & 0.04 & 0.04 & 0.01 \\
\hline \multirow{2}{*}{ Waist Length Front/Height } & U.S. & 0.23 & 0.19 & 0.21 & 0.23 & 0.25 & 0.28 & 0.09 \\
\hline & Korea & 0.21 & 0.18 & 0.20 & 0.20 & 0.22 & 0.23 & 0.05 \\
\hline \multirow{2}{*}{ Waist Length Back/Height } & U.S. & 0.27 & 0.24 & 0.26 & 0.27 & 0.28 & 0.30 & 0.05 \\
\hline & Korea & 0.24 & 0.22 & 0.23 & 0.24 & 0.25 & 0.27 & 0.04 \\
\hline \multirow{2}{*}{ Waist Girth/height } & U.S. & 0.54 & 0.43 & 0.47 & 0.52 & 0.59 & 0.70 & 0.27 \\
\hline & Korea & 0.49 & 0.39 & 0.43 & 0.48 & 0.53 & 0.63 & 0.23 \\
\hline \multirow{2}{*}{ Hip Girth / Height } & U.S. & 0.67 & 0.57 & 0.62 & 0.66 & 0.72 & 0.83 & 0.25 \\
\hline & Korea & 0.60 & 0.54 & 0.57 & 0.59 & 0.62 & 0.66 & 0.12 \\
\hline \multirow{2}{*}{ BMI } & U.S. & 0.27 & 0.19 & 0.22 & 0.25 & 0.30 & 0.39 & 0.20 \\
\hline & Korea & 0.23 & 0.18 & 0.20 & 0.22 & 0.25 & 0.29 & 0.11 \\
\hline
\end{tabular}

* Note: Range=value of Percentile 95- value of Percentile 05

\section{Conclusion and Implication}

This research is to provide feasible data for application in American and Korean apparel company which are related to apparel manufacturing and designing. This data was developed by analyzing information on Korean and American body sizes obtained from "Size USA Project" and "Size Korea Project".

The conclusions of this research were as follows.

First, we determined that American female measurements such as heights, weight, lengths, circumferences and drop values(except for Shoulder 
Yi Kyonghwa - Cynthia L. Istook / A Korean-American Comparative Study of 3D Scanned Female Anthropometric Data

$<$ Table 8> Standard values of Korean females vs. American females in computed values

\begin{tabular}{|c|c|c|c|c|c|c|}
\hline \multirow{2}{*}{ Computed values } & \multicolumn{2}{|c|}{ US } & \multicolumn{2}{|c|}{ Korean } & \multirow{2}{*}{$\begin{array}{l}\text { US } \\
(\%)\end{array}$} & \multirow{2}{*}{$\begin{array}{c}\text { Korean } \\
(\%)\end{array}$} \\
\hline & Mean & S.D. & Mean & S.D. & & \\
\hline Hip Girth-Bust Girth & 5.94 & 6.55 & 3.13 & 11.19 & 100 & 52.7 \\
\hline Hip Girth -Waist Girth & 22.21 & 6.06 & 16.41 & 12.22 & 100 & 73.9 \\
\hline Bust Girth -Waist Girth & 16.27 & 4.85 & 13.28 & 4.09 & 100 & 81.6 \\
\hline Back Neck Point Height/Height & 0.86 & 0.01 & 0.84 & 0.01 & 100 & 97.7 \\
\hline Waist Height/Height & 0.62 & 0.02 & 0.62 & 0.01 & 100 & 100.0 \\
\hline Hip Height/Height & 0.50 & 0.04 & 0.48 & 0.01 & 100 & 96.0 \\
\hline Crotch Height/Height & 0.45 & 0.02 & 0.44 & 0.01 & 100 & 97.8 \\
\hline Knee Height/Height & 0.27 & 0.01 & 0.26 & 0.01 & 100 & 96.3 \\
\hline Ankle Height/Height & 0.04 & 0.01 & 0.03 & 0.00 & 100 & 75.0 \\
\hline Waist Length Front/Height & 0.23 & 0.03 & 0.21 & 0.01 & 100 & 91.3 \\
\hline Waist Length Back/Height & 0.27 & 0.02 & 0.24 & 0.01 & 100 & 88.9 \\
\hline Waist Girth/height & 0.54 & 0.09 & 0.49 & 0.07 & 100 & 90.7 \\
\hline Hip Girth /Height & 0.67 & 0.08 & 0.60 & 0.04 & 100 & 89.6 \\
\hline $\mathrm{BMI}$ & 0.27 & 0.06 & 0.23 & 0.03 & 100 & 85.2 \\
\hline
\end{tabular}

Slope) were significantly larger than Korean female measurements. Using the BMI, we determined that American women had a distinct tendency towards being overweight.

Second, from the results of drops and ratios comparison between the two countries, we determined that (1) American women's body figures were more curved than Korean women; (2) American women had slightly shorter head lengths, higher hip heights, and longer leg lengths for their height compared to Korean woman; (3) Korean women's back shapes were flatter than American women and (4) BMI values indicated American women were more overweight than Korean women.

In conclusion, we could determine that American female measurements such as heights, weight, lengths, circumferences and drop values except for "Shoulder Slope" were significantly larger than Korean female measurements. And we can verify American women had a distinct tendency towards obesity.

\section{Reference}

1) CAESAR uses latest laser technology, Retrieved 2012. 9. 27, from http://www. sae.org/technicalcommittees/caelaser.htm.

2) US Army Natick Research, Development and Engineering Center(1989), 1988 Anthropometric Survey of U.S. Army Personnel: Methods and Summary Statistics, US Army.

3) $[\mathrm{TC}]^{2}$ (2004), The National Sizing Survey, Women: Body Measurements and Data Analysis Reports on the U.S. Population, $[\mathrm{TC}]^{2}$ Cary, NC, USA.

4) KS K 0050(2009), Sizing Systems for Elderly Women's Garments, Seoul, KSA, Korea.

5) KS K 0051(2009), Sizing Systems for Female Adult's Garments, KSA, Seoul, Korea.

6) Korean Agency for Technology \& Standards (2004), The $5^{\text {th }}$ Size Korea Survey Final Report. KATS Gwacheon, Korea.

7) ISO 3637(1990), Size designation of clothes 
- Women's and girls' Outwear Garments, ISO; Geneva, Switzerland.

8) ISO 7250(1996), Basic Human Body Measurements for Technological Design, ISO; Geneva, Switzerland.

9) ISO 8559(1989), Garment Construction and Anthropometric Surveys - Body Dimensions, ISO; Geneva, Switzerland.

10) ISO TR 10652(1991), Standard Sizing Systems for Clothes, ISO; Geneva, Switzerland.

11) ASTM D 5219(1999), Standard Terminology Relating to Body Dimension for Apparel Sizing, ASTM International, West Consho hocken, PA.

12) ASTM D 5585(1995), Standard Tables of Body Measurements for Adult Female Misses Figure Type, Size 2 to 20, ASTM International, West Conshohocken, PA.

13) ASTM D 7022(2004), Standard Terminology Relating to Apparel, ASTM International, West Conshohocken, PA.
14) ASTM D 5586(1995), Standard Tables of Body Measurements for Women Aged 55 and Older(All Figure Types), ASTM International, West Conshohocken, PA.

15) PS 42-70(1970), Body Measurements for the Sizing of Women's Patterns and Apparel, ASTM International, West Conshohocken, PA.

16) KS K 7003(2004), Garment Construction and Anthropometric Surveys-Body Dimensions, KSA, Seoul, Korea.

17) Yi Kyonghwa, et. al(2007), "Comparative Analysis of Korean and American Body Sizes \& Shapes using 3D Scanned Anthropometric Data, Journal of Korean Society of Clothing \& Textiles, 31(6), pp.891-901.

Received (Jun. 12, 2013)

Revised (Jun. 20, 2013, Jun. 29, 2013)

Accepted (Jul. 3, 2013) 